\title{
Expectativas de roles futuros de estudiantes universitarios en Chile
}

Future role expectations of undergraduate Chilean students

Recibido: julio 27 de 2010 | Revisado: enero 12 de 2011 | Aceptado: diciembre 12 de 2011

\author{
NUREYA ABARCA ** \\ Pontificia Universidad Católica de Chile \\ NAISA GORMAZ ${ }^{* * *}$ \\ Escuela de Psicología, Universidad Diego Portales \\ Pontificia Universidad Católica de Chile \\ PEDRo I. LeIvA ${ }^{* * * *}$ \\ Facultad de Economía y Negocios, \\ Universidad de Chile
}

\section{RES UMEN}

Este estudio examina las expectativas de roles futuros de 522 jóvenes universitarios en Santiago de Chile. Para esto, se utilizó la adaptación de un instrumento que evalúo las expectativas personales de hombres y mujeres sobre distintos roles que pueden ejercer en la vida: laboral, marital, parental y cuidado del hogar (The Life Role Salience Scales [LRSS]; Amatea, Cross, Clark \& Bobby, 1986). Se evaluaron el nivel de Conservadurismo Valórico (Contreras \& Plaza, 2004) y los datos sociodemográficos. Los análisis de regresión mostraron una preferencia masculina mayor en roles laboral y marital en comparación con las mujeres. El Conservadurismo Valórico influyó en expectativas de roles marital, parental y cuidado del hogar, mas no en el rol laboral.

Palabras clave autores

Expectativas de rol, rol laboral, rol marital, rol parental, jóvenes universitarios.

Palabras clave descriptores

Psicología social, investigación cuantitativa, pruebas psicológicas.

Para citar este artículo. Abarca, N., Gormaz, \& Leiva, P. I. (2012). Expectativas de roles futuros de estudiantes universitarios en Chile. Universitas Psychologica, 11(2), 405-414.

Naturaleza del artículo: proveniente de una investigación original.

** Escuela de Administración, Pontificia Universidad Católica de Chile. La correspondencia relativa a este artículo debe ser enviada a Nureya Abarca, Av. Vicuña Mackenna 4860, Macul, Santiago de Chile.E-mail: nabarca@uc.cl

**** Escuela de Psicología, Universidad Diego Portales. Escuela de Psicología, Pontificia Universidad Católica de Chile. E-mail: ngormaz@uc.cl. ResearcherID Gormaz, N. F-3311-2012.

wnew Departamento de Administración. E-mail: pleivan@unegocios.cl. ResearcherID Leiva, P. F-3475. 2012.

\section{A B S T R A C T}

This study reviews the future roles expectations in a sample of 522 young undergraduate students in Santiago, Chile. In order to do that it was administered the adaptation of the instrument (The Life Role Salience Scales [LRSS]; Amatea, Cross, Clark \& Bobby, 1986) that assessed men's and women's personal expectations concerning life roles: occupational, marital, parental and homecare roles. As well the level of Valoric Conservatism (VC) was measure (Contreras \& Plaza, 2004), and socio-demographic data. Regression analysis showed a male's bigger preference for work and marital roles than women. VC influenced the expectations for marital, parental and home-care roles, but it didn't influenced work role.

Key words authors

Role expectations, occupational role, parental role, marital role, undergraduate students.

Key words plus

Social psychology, quantitative research, psychological tests. 
Las personas jóvenes que aún no han empezado a trabajar se encuentran en un período de cambio y de búsqueda de su identidad que precede a su posicionamiento en roles de adulto (Duarte, 2000). Arnett (2000) fija dicha etapa entre los 18 y 25 años de edad. Actualmente, en países industrializados, la mayoría de los jóvenes adultos dedica estos años a invertir y preparar su participación en dos roles muy relevantes en su vida: el trabajo y la familia (Cinamon, 2006).

Se ha señalado que el nivel de importancia y compromiso que el joven adulto le atribuya a sus futuros roles, así como el equilibrio que espera alcanzar entre los distintos roles, son importantes precursores de la habilidad para manejar estos roles una vez que los ejerza (Kerpelman \& Schvaneveldt, 1999).

En el pasado, el nivel de importancia y compromiso del rol y las expectativas acerca de las prioridades de cada rol estaban determinados primariamente por el género de la persona. Pero actualmente, los jóvenes adultos anticipan un compromiso más variado con respecto a los distintos roles en el trabajo y la familia. Algunas investigaciones muestran que los hombres tienden a ser más tradicionales que las mujeres en sus expectativas sobre roles en el trabajo y familia (Beutel \& Marini, 1995), privilegiando el rol laboral por sobre el marital, en tanto las mujeres valorarían más el rol parental que el laboral (Sanders, 2004). Sin embargo, otras investigaciones muestran resultados diferentes señalando que las expectativas sobre rol marital se han vuelto más igualitarias. Las mujeres jóvenes valoran tanto el rol de trabajadora como de madre, y hombres y mujeres jóvenes dan similar importancia a identidades anticipadas maritales, laborales (Blakemore, Lawton \& Vartanian, 2005) y parentales (Olavarría, 2001). Esta discrepancia entre distintos estudios indica la necesidad de entender más sobre las expectativas de jóvenes adultos y los roles que asumirán en su vida laboral y familiar.

\section{Antecedentes internacionales}

Las actitudes y preferencias hacia la familia y el trabajo en los jóvenes se han relacionado con la anticipación de conflictos que pueden ocurrir en el futuro entre los distintos roles. Tener que manejar los conflictos que provienen de roles que compiten entre sí, ha llegado a ser una experiencia frecuente en hombres y mujeres en las sociedades industrializadas. Una fuente importante de estrés en las personas que tratan de resolver estos conflictos se encuentra en la naturaleza de las expectativas sobre su vida personal, familiar y laboral (Rapoport, Bailyn, Fletcher \& Pruitt, 2002). Hombres y mujeres frecuentemente se encuentran enfrentados a conflictos que tienen relación con metas futuras (Cinamon \& Rich, 2002a). Sin embargo, un metaanálisis realizado por Shockley y Singla (2011) reportó que el conflicto entre los roles laboral y familiar impactaba la satisfacción general en mayor medida en mujeres que en hombres.

Las expectativas de las mujeres de poder comprometerse en el futuro, tanto con su carrera como con la familia, se ven en dificultades por expectativas que se observan en la sociedad. Por un lado, tanto hombres como mujeres consideran que los roles primordiales de una esposa son el cuidado y responsabilidad de los niños (Olavarría, 2001) y, por otro lado, los hombres muestran menor preferencia por asumir los roles domésticos y familiares (Friedman \& Weissbrod, 2005).

Por tanto, al parecer, la anticipación de estos conflictos puede jugar un papel importante en los planes de desarrollo de carrera de los jóvenes adultos (Cinamon, 2006). Algunos podrán reducir sus aspiraciones profesionales en favor de la familia o viceversa, para evitar las consecuencias negativas del conflicto, lo que, como ya se señaló, parece ser más frecuente en el caso de las mujeres que de los hombres. En general, las mujeres presentan una tendencia a presentar desarrollos de carrera más lentos que los hombres, influidos por salidas y retornos al mercado laboral debido a los embarazos (Eagly \& Carli, 2007). Algunos estudios han sugerido que este desarrollo de carrera más lento en el caso de la mujer se comienza a gestar en la vida universitaria, en donde se producirían cambios en las actitudes de hombres y mujeres con respecto a los roles que ejercerán en el futuro (Bryant, 2003; Cinamon \& Rich, 2002b). Es así como las mujeres 
lograrían desarrollar su rol femenino a través del énfasis asignado a la pertenencia a una comunidad y a las relaciones sociales, en oposición a los hombres, quienes cumplirían con el estereotipo masculino centrándose en el cumplimiento de tareas y en el logro de sus metas (Gaeddert, 1985).

Los roles que se espera que hombres y mujeres cumplan en el futuro generan conflictos en ambos. Dado que los hombres también experimentan una fuerte presión en términos de sus decisiones futuras, metas y expectativas en una dirección diferente a la de las mujeres, se ven muy afectados por las normas sociales que les imponen disfrazar sus emociones, mostrarse seguros, tener éxito y ser competitivos (Good \& Mintz, 1990). Debido a la complejidad presente en los roles que deben asumir hombres y mujeres, sería pertinente ir más allá de la simple dicotomía trabajo-familia y abordar las suposiciones y expectativas subyacentes acerca de los roles en la familia y en el trabajo remunerado que tienen los jóvenes. Dichas suposiciones podrían moldear tanto los lugares de trabajo como las instituciones sociales.

A pesar del nivel de importancia y compromiso que tienen las expectativas de los jóvenes adultos en las futuras combinaciones de roles en cuanto a su desempeño futuro en su vida laboral y familiar, el conocimiento que se tiene al respecto aún es limitado.

\section{Antecedentes en Chile}

Chile es considerado un país tradicional en lo que se refiere a roles de género (Abarca \& Majluf, 2003). Según el Ministerio del Trabajo (2010), presenta la participación femenina laboral más baja de América Latina (40\%). Tradicionalmente, en Chile las mujeres tienden a preferir roles relacionados con el cuidado de la casa y la familia, mientras que los hombres en general prefieren dedicarse a su carrera. Contreras y Plaza (2004) señalan que Chile aparece como un país poco proclive al trabajo de la mujer fuera del hogar, destacándose principalmente los costos familiares que este implica.

Las actitudes "machistas" por parte del entorno que rodea a la mujer y el nivel de Conservadurismo
Valórico (CV) que presentarían ellas mismas, son los dos factores que incidirían en el bajo nivel de inserción laboral de las mujeres. Adicionalmente, la Encuesta Nacional del Bicentenario UC-Adimark (Pontificia Universidad Católica de Chile \& Adimark/GfK, 2007) reporta que aproximadamente la mitad de los encuestados considera que el hombre es quien debe trabajar y la mujer debe quedarse en la casa. Mientras que un porcentaje mayor considera que la familia se descuida cuando la mujer trabaja de tiempo completo.

En Chile, la presencia femenina en carreras tradicionalmente dominadas por hombres se ha incrementado considerablemente -por ejemplo, según el Consejo Superior de Educación de Chile (CSE) constituían un $16.6 \%$ de los estudiantes de las carreras de Administración y Comercio en el 2006-. Lo anterior nos permite inferir que nos encontramos ante un escenario de gran dinamismo. En este contexto, es interesante investigar si las percepciones con respecto a formar una familia y dedicarse a la vida laboral, difieren según género y nivel de CV. Un estudio en esta línea contribuiría al desarrollo de conocimientos en esta área, la cual consta de pocas publicaciones en Chile.

El presente estudio evalúa las expectativas personales de estudiantes universitarios chilenos del área de la gestión y administración de empresas, sobre los distintos roles que les tocará ejercer: laboral, marital, parental y de cuidado del hogar. Si bien esta muestra no es representativa de la población universitaria chilena, es de gran relevancia debido a que cursan carreras que los ubicarán en puestos de trabajo de nivel directivo medio y alto. Adicionalmente, esta muestra de estudiantes pertenece a carreras que tienden a concentrar un mayor número de hombres que de mujeres.

Las hipótesis que se plantean en la presente investigación son:

1. El nivel de importancia y compromiso que los estudiantes universitarios otorgan a su futuro rol varía según el género.

2. El nivel de CV varía de acuerdo con el género.

3. Hay diferencias significativas en las preferencias hacia roles futuros según el nivel de CV. 
4. Existen diferencias significativas en los niveles de CV según expectativas sobre matrimonio e hijos/as.

5. Existen diferencias significativas respecto al nivel de importancia y compromiso con los futuros roles (laboral, marital, parental y de cuidado del hogar), según las expectativas sobre matrimonio e hijos/as y estado civil.

\section{Método}

\section{Participantes}

La muestra definitiva fue de 522 estudiantes de una universidad en la ciudad Santiago de Chile, quienes reportaron cursar los últimos años en las carreras de Ingeniería Comercial (47.3\%), Ingeniería Industrial (24.3\%), cursos de Master of Business Administration (MBA) $(25.4 \%)$ y otros (2.1\%). De ellos, el $32.2 \%$ correspondía a mujeres y el $67.8 \%$, a hombres; el promedio de edad fue de 24.5 años $(D E=4.2)$; y el $88 \%$ estaba constituido por solteros y el $12 \%$, por casados.

\section{Instrumento}

\section{Escala Roles de Vida (LRSS)}

Creada por Amatea, Cross, Clark y Bobby (1986), la escala evalúa el nivel de importancia y compromiso con las expectativas de rol con las que las personas se identifican respecto de cuatro roles: marital, parental, cuidado de la casa y laboral. Cada expectativa de rol fue evaluada por medio de 10 ítems. Ejemplos de ellos son: "Tener un matrimonio exitoso es lo más importante en mi vida", "Espero dedicar una cantidad de tiempo y energía considerable a la crianza de mis hijos/as", "Espero estar muy involucrado/a en cuidar mi casa y hacerla atractiva" y "Espero dedicar una cantidad considerable de mi tiempo a construir mi carrera y desarrollar las habilidades necesarias para avanzar en ella", respectivamente para cada rol. El formato de respuesta es de tipo Likert, con 5 puntos $(1=$ muy en desacuerdo y $5=$ muy de acuerdo). En el presente estudio, se uti- lizó la versión adaptada del LRSS (Abarca, 2004), que se evaluó en una muestra piloto.

Un análisis factorial de los 40 ítems demostró que los resultados de las escalas en esta muestra poseían baja validez de constructo. Por lo mismo, se seleccionaron los cinco ítems de cada rol que reportaron la mayor correlación ítem-escala corregido. El análisis factorial con los 20 ítems seleccionados evidenció la estructura de cuatro factores hipotetizada. Los índices de confiabilidad obtenidos por cada subescala se reportan junto a los estadísticos descriptivos y las correlaciones entre las variables del estudio en la Tabla 1.

\section{Indicadores de CV}

Contreras y Plaza (2004) propusieron cuatro ítems para evaluar el nivel de actitudes machistas y cinco para el CV por medio de una escala Likert, con 5 puntos $(1=$ muy en desacuerdo y $5=$ muy de acuerdo). El análisis factorial de estos indicadores reveló la presencia de un factor que agrupaba a siete ítems. Por tanto, se decidió trabajar solo con la escala de CV que reportó un buen índice de confiabilidad (alfa de Cronbach $=0.79$ ). Dado que los ítems se presentan invertidos, por ejemplo "Es una buena idea para una pareja que tiene intención de casarse, vivir juntos primero", el promedio de la escala fue reversado con el objeto de facilitar la comprensión de los resultados, de modo que mayor puntaje implica mayor nivel de conservadurismo.

\section{Expectativas de matrimonio y número de hijos/as}

Tres preguntas evaluaron las expectativas de matrimonio en razón de la cantidad de años en que se espera contraer matrimonio. Por lo mismo, mayor puntaje en esta variable indica expectativa de matrimonio más tardía. También se evaluaron las expectativas de número de hijos/as de los participantes, consultando la cantidad de años en que espera tener hijos/as y cuántos.

\section{Datos sociodemográficos}

Se consultó el género, la edad, el estado civil y el número de hijos/as actual de los participantes. 
TABLA 1

Estadísticos descriptivos e intercorrelaciones

\begin{tabular}{|c|c|c|c|c|c|c|c|c|c|c|c|}
\hline & Media & $\mathrm{DE}$ & 1 & 2 & 3 & 4 & 5 & 6 & 7 & 8 & 9 \\
\hline 1. Género & 0.32 & 0.47 & -. & & & & & & & & \\
\hline 2. Edad & 24.48 & 4.2 & $-0.14^{* * *}$ & -. & & & & & & & \\
\hline 3. Estado Civil & 0.12 & 0.33 & $-0.09^{*}$ & $0.64^{* *}$ & -. & & & & & & \\
\hline 4. Número de Hijos/as & 0.15 & 0.58 & -0.11 & $0.57^{* *}$ & $0.57^{* *}$ & .- & & & & & \\
\hline 5. CV & 3.1 & 0.89 & 0.02 & -0.06 & 0.06 & $0.09^{*}$ & 0.79 & & & & \\
\hline 6. Rol Marital & 3.96 & 0.76 & $-0.11^{* *}$ & 0.08 & $0.17^{* *}$ & $0.1^{*}$ & $0.38^{* *}$ & 0.85 & & & \\
\hline 7. Rol Parental & 4.5 & 0.52 & 0.01 & 0.05 & $0.1^{*}$ & $0.12^{*}$ & $0.34^{* *}$ & $0.56^{* *}$ & 0.82 & & \\
\hline 8. Rol Cuidado del Hogar & 3.51 & 0.7 & 0.01 & $-0.1^{*}$ & -0.06 & -0.02 & $0.18^{* *}$ & $0.3^{* *}$ & $0.37^{* *}$ & 0.83 & \\
\hline 9. Rol Laboral & 3.98 & 0.49 & -0.11 & 0.01 & -0.01 & 0.02 & -0.03 & -0.06 & -0.03 & $0.17^{* *}$ & 0.77 \\
\hline
\end{tabular}

$* p<0.05$. ** $p<0.01$.

Nota. Coeficientes de alfa de Cronbach se reportan en la diagonal. Género: $0=$ hombre $1=$ mujer; Estado Civil: $0=$ soltero $1=$ casado.

Fuente: elaboración propia.

\section{Procedimiento}

Tras conseguir el consentimiento informado de parte de los estudiantes, se administró el instrumento al principio o término de una clase lectiva durante el segundo semestre de 2004, en los cursos de las carreras ya señaladas. A los participantes se les informó que se trataba de un estudio sobre expectativas de trabajo y familia, que su participación era completamente voluntaria y anónima, y que no representaba ningún riesgo para su salud psíquica y física.

\section{Resultados}

Hipótesis 1: diferencias en expectativas de roles según género

Se realizaron cuatro regresiones simples considerando género como variable independiente y con cada una de las expectativas de rol como variables dependientes. Edad, estado civil y número de hijos/ as fueron incorporados al modelo como variables control. Los resultados apoyan la hipótesis solo para rol marital, aun incluyendo a las variables control, y para el rol laboral solo cuando no se incluyeron estas últimas.
Género explicó significativamente $1 \%$ adicional de la varianza de la expectativa de rol marital, controlando por edad, estado civil y número de hijos/as. El modelo completo explicó $4 \%$ de la varianza de esta expectativa de rol $\left(r^{2}=0.04 ; F(4.508)=5.28\right.$, $p=0.00$ ), pero solo estado civil y género resultaron significativos. Las personas casadas reportan mayor nivel de expectativas de este rol $(\beta=0.19$, $p=0.00,95 \%$ IC $[0.08,0.31] ; M_{\text {casados }}=4.630$, $\left.D E=0.57 ; M_{\text {solteros }}=3.90, D E=0.77\right)$. Por otro lado, los hombres reportan mayor nivel de expectativas de rol marital, $(\beta=-0.10, p=0.02,95 \%$ IC $[-0.19,-0.01] ;\left(M_{\text {hombres }}=4.01, D E=0.72\right.$; $\left.\mathrm{M}_{\text {muieres }}=383, \mathrm{DE}=0.84\right)$.

Respecto de rol laboral, cuando se incluyeron las variables control, el modelo no resultó significativo $(F(4.508)=1.62$, n.s.). Sin embargo, sin las variables control, género explica $1 \%$ de la varianza $\left(r^{2}=0.01\right)$. Los hombres reportan un mayor nivel $(F(1.514)=6.03, p=0.01, \beta=-0.11, p=0.01$, $95 \%$ IC [-0.19, - 0.04$] ; M_{\text {hombres }}=4.02, D E=0.48$; $\mathrm{M}_{\text {muieres }}=3.91, \mathrm{DE}=0.51$ ).

Sin embargo, no observamos variaciones según género para rol parental $(F(4.508)=2.27$, n.s. $)$, $(F(1.514)=0.34$, n.s. con y sin variables control, respectivamente), ni para cuidado del hogar $(F(4.508)=1.493$, n.s; $F(1.514)=0.03$, n.s. $)$. 


\section{Hipótesis 2: nivel de importancia y compromiso del futuro rol según $\mathrm{CV}$}

Los resultados no entregan apoyo a esta hipótesis. Aun cuando el modelo resulta significativo $\left(r^{2}=0.03 ; F(4.508)=4.476, p=0.00\right)$, esto se debe al efecto de edad y número de hijos/as sobre el nivel de $\mathrm{CV}$. Mientras mayor edad menor es el grado de $\operatorname{CV}(\beta=-0.21, p=0.00,95 \%$ IC $[-0.32,-0.12])$, y a más hijos/as mayor nivel de $\mathrm{CV}(\beta=0.16, p=0.01$, $95 \%$ IC $[0.05,0.27])$. Estado civil no presentó un efecto significativo. Finalmente, $\mathrm{CV}$ no varió significativamente según género ( $\beta=0.01$, n.s., $95 \%$ IC $[-0.07,0.10])$.

\section{Hipótesis 3: diferencias en expectativas de roles según $\mathrm{CV}$}

Se regresó cada una de las expectativas de rol en $\mathrm{CV}$, controlando por género, edad, estado civil y número de hijos/as. Los resultados apoyan a la hipótesis para expectativa de rol marital, parental y de cuidado del hogar, pero no para rol laboral. El CV explicó $13.9 \%$ adicional de la varianza de rol marital respecto del $4 \%$ explicado por género $\left(r^{2}=0.18 ; F(5.507)=22.06, p=0.00\right)$. Edad $y$ número de hijos/as no tuvo efecto significativo sobre la expectativa de rol marital. Sin embargo, los hombres reportaron mayores expectativas de rol marital $(\beta=-0.11, p=0.01,95 \%$ IC [-0.19, -0.03]), así como los casados $(\beta=0.15, p=0.01$, $95 \%$ IC $[0.06,0.24])$. En relación con $C V$, mayores puntajes se asociaron con mayores niveles en esta expectativa ( $\beta=0.38, p=0.00,95 \%$ IC [0.30, 0.46]).

En relación con el rol parental, CV explicó $11 \%$ adicional de su varianza una vez considerados las variables control $\left(r^{2}=0.12 ; F(5.507)=14.31\right.$, $p=0.00$ ). Aun cuando ninguna de las variables control se asoció con rol parental, mayores niveles de CV se asociaron con mayores niveles de esta expectativa $(\beta=0.33, p=0.00,95 \%$ IC $[0.25,0.41])$. Similarmente, pero con menor magnitud, CV explicó $3 \%$ de la varianza del rol del cuidado del hogar, por encima de las variables control $\left(r^{2}=0.04\right.$; $F(5.507)=4.16, p=0.00)$. Las personas con mayor nivel de CV presentaron mayores expectativas de rol del cuidado del hogar $(\beta=0.17, p=0.00,95 \%$ IC $[0.08,0.26])$.

Para rol laboral, ni las variables control ni CV explicaron esta expectativa $(F(5.507)=0.21$, n.s.; $F(1.520)=0.45$, n.s. con y sin variables control, respectivamente).

\section{Hipótesis 4: CV según expectativas sobre matrimonio e hijos/as}

Para poner a prueba las Hipótesis 4 y 5 , fue necesario excluir del análisis los participantes que reportaron estar ya casados (66), no querer casarse (8) y tener hijos/as (43), debido a que estas expectativas podrían estar cumplidas. Los estadísticos descriptivos e intercorrelaciones para esta submuestra de 431 participantes revelaron que las variables "cuándo casarse" y "cuándo tener hijos/as" se encontraban altamente correlacionadas (véase Tabla 2). Para evitar multicolinearidad, contrastamos estas hipótesis incluyendo en los modelos de regresión solo "cuándo casarse" y "cuántos hijos/as tener". Las variables edad y género fueron incluidas como variables control.

La Hipótesis 4 recibe apoyo de los datos controlando por género y edad. Las expectativas de matrimonio y de número de hijos/as explicaron 29 \% de la varianza de CV adicionales al $2.2 \%$ de la varianza explicada por género y edad $\left(r^{2}=0.31\right.$; $F(4.424)=40.05, p=0.00$ ). Aun cuando género no influye significativamente en $\mathrm{CV}$, las personas mayores reportaron menor nivel de $\mathrm{CV}(\beta=-0.18$, $p=0.00,95 \%$ IC $[-0.27,-0.08])$. Quienes esperan casarse más tardíamente, presentaron menos $\mathrm{CV}$ $(\beta=-0.24, p=0.00,95 \%$ IC $[-0.33,-0.14])$. Lo contrario sucede con quienes desean tener más hijos/as $(\beta=0.44, p=0.00,95 \%$ IC $[0.35,0.52])$.

\section{Hipótesis 5: expectativas sobre matrimonio e hijos/as y nivel de expectativas de roles}

Los resultados apoyan esta hipótesis para rol marital, parental y cuidado del hogar cuando se controló por edad y género. Pero solo al excluir las variables control para rol laboral. Las expectativas 
TABLA 2

Estadísticos descriptivos e intercorrelaciones submuestra de solteros y sin hijos/as

\begin{tabular}{|c|c|c|c|c|c|c|c|c|c|c|c|c|}
\hline & Media & $D E$ & 1 & 2 & 3 & 4 & 5 & 6 & 7 & 8 & 9 & 10 \\
\hline 1. Género & 0.34 & 0.47 & .. & & & & & & & & & \\
\hline 2. Edad & 23.45 & 2.66 & -0.07 & -. & & & & & & & & \\
\hline $\begin{array}{l}\text { 3. Expectativa de } \\
\text { Matrimonio }\end{array}$ & 2.48 & 0.72 & $-0.15^{* *}$ & $-0.41^{* *}$ & -. & & & & & & & \\
\hline $\begin{array}{l}\text { 4. Expectativa de } \\
\text { Hijos/as }\end{array}$ & 2.85 & 0.71 & -0.08 & $-0.40^{* *}$ & $0.70^{* *}$ & -. & & & & & & \\
\hline $\begin{array}{l}\text { 5. Expectativa de } \\
\text { Número de Hijos/as }\end{array}$ & 3.46 & 1.44 & -0.06 & $-0.17^{* *}$ & $-0.20^{* *}$ & $-0.27^{* *}$ & .- & & & & & \\
\hline 6. $\mathrm{CV}$ & 3.12 & 0.89 & -0.00 & $-0.15^{* *}$ & $-0.25^{* *}$ & $-0.26^{* *}$ & $0.52^{* * *}$ & & & & & \\
\hline 7. Rol Marital & 3.94 & 0.76 & $-0.12^{*}$ & -0.03 & $-0.32^{* * *}$ & $-0.30^{* *}$ & $0.31^{* *}$ & $0.37^{* *}$ & 0.88 & & & \\
\hline 8. Rol Parental & 4.50 & 0.50 & 0.03 & -0.02 & $-0.28^{* *}$ & $-0.38^{* * *}$ & $0.33^{* *}$ & $0.31^{* *}$ & $0.54^{* *}$ & 0.80 & & \\
\hline $\begin{array}{l}\text { 9. Rol Cuidado del } \\
\text { Hogar }\end{array}$ & 3.53 & 0.68 & -0.01 & 0.03 & $-0.19 * *$ & $-0.24^{* * *}$ & $0.19^{* * *}$ & $0.16^{* * *}$ & $0.33^{* * *}$ & $0.43^{* *}$ & 0.82 & \\
\hline 10. Rol Laboral & 3.98 & 0.49 & $-0.10^{*}$ & -0.01 & $0.11^{*}$ & $0.11^{*}$ & -0.06 & -0.03 & -0.05 & -0.02 & $0.18^{* * *}$ & 0.78 \\
\hline
\end{tabular}

$N=431 . * p<0.05 . * * p<0.01$.

Nota. Coeficientes de alfa de Cronbach se reportan en la diagonal. Género: $0=$ hombre $1=$ mujer

Fuente: elaboración propia.

de matrimonio y de número de hijos/as explicaron el $19 \%$ adicional de la varianza del rol marital. El modelo con las cuatro variables explicó $20 \%$ de la varianza total de esta expectativa de rol $\left(r^{2}=0.20\right.$; $F(4.424)=26.75, p=0.00$ ). En línea con la Hipótesis 1 , los hombres muestran mayor expectativa de rol $(\beta=-0.17, p=0.00,95 \%$ IC $[-0.26,-0.08])$. En esta submuestra, edad se relacionó negativamente con rol marital $(\beta=-0.17, p=0.00,95 \%$ IC $[-0.26,-0.06])$. Cuando las personas esperan casarse más tardíamente, presentan un menor nivel de expectativas en este rol $(\beta=-0.37, p=0.00$, $95 \%$ IC $[-0.47,-0.27])$, y lo contrario ocurre cuando las personas desean tener más hijos/as $(\beta=0.20$, $p=0.00,95 \%$ IC $[0.11,0.29])$.

Para rol parental, género y edad no explican significativamente la varianza de las expectativas de este rol, mientras que las dos variables hipotetizadas explican $16 \%$ de la varianza $\left(r^{2}=0.16\right.$; $F(4.424)=20.51, p=0.00$ ). Las personas que esperan casarse más tardíamente, presentan menor nivel de expectativas en este rol; $(\beta=-0.26, p=0.00$, $95 \%$ IC $[-0.36,-0.16])$, y en la medida en que desean tener más hijos/as tienen mayor expectativa $(\beta=0.27, p=0.00,95 \%$ IC $[0.17,0.36])$.
Algo similar sucede con el rol cuidado de hogar: solo las dos variables hipotetizadas explican $6 \%$ de la varianza de esta expectativa $\left(r^{2}=0.06\right.$; $F(4.424)=6.50, p=0.00)$. Quienes esperan casarse más tardíamente, presentan un menor nivel de expectativas en este rol $(\beta=-0.16, p=0.00,95 \%$ IC $[-0.27,-0.05])$, y quienes desean tener más hijos/ as presentan mayor expectativa $(\beta=0.15, p=0.00$, $95 \%$ IC $[0.05,0.25])$.

Para el rol laboral, al controlar por género y edad, el modelo no es significativo $(F(4.424)=2.47$, n.s.). Sin embargo, sin estas variables, las dos variables hipotetizadas explican $1.5 \%$ de la varianza de esta expectativa de rol $\left(r^{2}=0.02 ; F(2.426)=3.23\right.$, $p=0.04)$. Sin embargo, solo "cuándo casarse" se relacionó significativamente con las expectativas en este rol $(\beta=0.11, p=0.03,95 \%$ IC [0.02, 0.20]), no así el número de hijos/as ( $\beta=-0.04$, n.s. $95 \%$ IC $[-0.13,0.05)$.

\section{Discusión}

Debido a los cambios experimentados en las sociedades industriales, los jóvenes actualmente deben combinar los roles laborales y de familia. En el tránsito hacia la adultez se deben explorar diferen- 
tes posibilidades respecto al trabajo y las relaciones familiares antes de tomar decisiones de largo plazo (Cinamon, 2005). En el presente estudio se investigaron las expectativas de roles futuros marital, parental, de cuidado del hogar y laboral en jóvenes universitarios chilenos en relación con el género, $\mathrm{CV}$ y expectativas de matrimonio e hijos/as.

Los resultados obtenidos mostraron una diferencia significativa según género en los roles laboral y marital. Así, los resultados obtenidos confirman en parte -evidencia anterior- el nivel de importancia y compromiso que dan los hombres a su rol laboral, pero son distintos pues revelan un mayor nivel con el rol marital, lo cual se atribuye más a menudo a las mujeres (Sanders, 2004). Otro estudio realizado en Chile obtuvo un resultado similar (McWhirter \& McWhirter, 2008): escolares adolescentes de estrato socieconómico medio-bajo presentaron expectativas ligeramente más bajas que los hombres respecto a casarse antes de los 25 años y tener hijos/as. En la misma línea, Chi-Ching (1995) examinó los efectos en Singapur de la carrera elegida y variables del ciclo vital (p. ej., matrimonio) y su influencia en la importancia atribuida a los roles laborales y familiares, encontrando, en el caso de las mujeres, que quienes dan alta importancia a su carrera están menos comprometidas con su rol parental y marital. Los hombres daban alta importancia al rol laboral, pero en concordancia con nuestro estudio, también mostraron altos puntajes en los roles parental y marital.

Una posible explicación de este paradójico resultado puede deberse a las características de las mujeres que eligen estudiar esta clase de carreras demandantes en su ejercicio profesional. Por tanto, podría suponerse que las mujeres estarían en capacidad de prever la necesidad de una gran dedicación a su carrera, lo cual puede relacionarse con la anticipación de posibles conflictos entre los futuros roles laborales y familiares. Dicha anticipación jugaría un rol central en sus planes de carrera, de modo que en este caso las mujeres reducirían momentáneamente sus aspiraciones familiares (Friedman \& Weissbrod, 2005; Niles \& Goodnough, 1996). En nuestro contexto cultural, donde el matrimonio suele estar unido a los hijos y donde se concibe a la mujer como la cuidadora principal (Caamaño, 2004), la situación anterior podría considerarse aún más como un obstáculo en el desarrollo de la carrera profesional.

Contrariamente a los resultados obtenidos por Contreras y Plaza (2004), no se encontraron diferencias de género en el $\mathrm{CV}$, lo cual podría deberse al rango etario y al nivel educacional de la muestra. Ambos elementos pueden estar relacionados con actitudes similares en hombres y mujeres.

En relación con las expectativas de matrimonio, quienes esperan casarse más tardíamente presentaron menor nivel de CV, expectativas de rol marital, parental y del cuidado del hogar, y un mayor nivel de expectativas de rol laboral. Lo anterior podría ir en línea con investigaciones que han señalado que las personas más jóvenes y educadas suelen ser más liberales que las personas de mayor edad (p. ej., Maureira, 2008), y que quienes esperan invertir en gran medida en su rol laboral no esperan casarse ni tener hijos pronto (Chi-Ching, 1995).

Por otra parte, CV presentó una relación positiva con las expectativas de rol marital, parental y de cuidado del hogar, no así con el rol laboral. Asimismo, se aprecia entre quienes desean tener más hijos/as, que presentan un mayor nivel de $\mathrm{CV}$, expectativas en su rol marital, parental y cuidado del hogar. Lo anterior apoyaría la importancia de considerar CV como un elemento que influye en el modo en que las expectativas de rol se manifiestan, de manera que se podría hipotetizar, por ejemplo, que las personas que adhieren a ideales más conservadores, independiente de su edad, tenderían a tener expectativas más acordes a concepciones más tradicionales de género relacionadas con querer casarse, tener hijos y preocuparse del lugar en que se vive. Por ejemplo, algunos estudios han mostrado que hombres jóvenes con actitudes más liberales hacia los roles de género esperan una distribución más igualitaria de las tareas domésticas y del cuidado de los hijos que hombres con actitudes más conservadoras. A su vez, mujeres jóvenes con actitudes más liberales esperan no ser las principales encargadas de los quehaceres domésticos y de crianza al casarse (Askari, Liss, Erchull, Staebell \& Axelson, 2010). 
Una de las limitaciones del presente estudio se refiere a la capacidad de generalización de los resultados a la población de jóvenes, debido a las características particulares de la muestra. Sin embargo, tal como se planteó en la introducción, se consideró que esta muestra es de gran relevancia para el estudio de personas con alto nivel educacional que se desempeñarán en cargos directivos asociados al mundo de los negocios. En el marco de las futuras investigaciones, se sugiere ampliar los hallazgos de esta investigación replicándolos en muestras de otras características y en otros contextos socioculturales. Otro ámbito relevante sería investigar sobre la percepción de posible conflicto entre los roles futuros. Y, finalmente, sería recomendable incluir la variable de $\mathrm{CV}$ en dichas investigaciones para explorar con mayor detalle su rol en las manifestaciones de las expectativas de rol.

\section{Referencias}

Abarca, N. (2004). Adaptación del instrumento LRSS. Manuscrito sin publicar, Escuela de Administración, Pontificia Universidad Católica de Chile, Santiago, Chile.

Abarca, N. \& Majluf, N. (2003). Women in management: A study of Chilean executives. Abante, 6, 55-81.

Amatea, E., Cross, E. G., Clark, J. \& Bobby, C. (1986). Assessing the work and family role expectations of career-oriented men and women: The life role salience scales. Journal of Marriage and the Family, 48, 831-838.

Arnett, J. (2000). Emerging adulthood: A theory of development from the late teens through the twenties. American Psychologist, 55, 469-480.

Askari, S. F., Liss, M., Erchull, M. J., Staebell, S. E. \& Axelson, S. J. (2010). Men want equality, but women don't expect it: Young adults' expectations for participation in household and child care chores. Psychology of Women Quarterly, 34(2), 243-252.

Beutel, A. \& Marini, M. (1995). Gender and values. American Sociological Review, 60, 436-448.

Blakemore, J., Lawton, C. \& Vartanian, L. (2005). I can't wait to get married: Gender differences in drive to marry. Sex Roles, 53 (5-6), 327-335.
Bryant, A. (2003). Changes in attitudes toward women's roles: Predicting gender-role traditionalism among college students. Sex Roles, 48, 131-142.

Caamaño, E. (2004). Conciliación de trabajo y vida familiar. Un análisis desde la perspectiva del derecho del trabajo chileno. Revista de Derecho, 16, 59-82.

Cinamon, R. (2005, August). Anticipated work-family conflict: Effects of attributions toward life role and self-efficacy. Paper presented at the Conference of the American Psychological Association, Washington, DC, USA.

Cinamon, R. (2006). Anticipated work-family conflict: Effects of gender, self-efficacy, and family background. The Career Development Quarterly, 54, 202-215.

Cinamon, R. \& Rich, Y. (2002a). Gender differences in attribution of importance to life roles: Implications for the work-family conflict. Sex Roles, 47, 531-541.

Cinamon, R. \& Rich, Y. (2002b). Profiles of attribution of importance to life roles: Implications for the work-family conflict. Journal of Counseling Psycho$\log y, 49,212-220$.

Chi-Ching, Y. (1995). The effects of career salience and life-cycle variables on perceptions of work-family interfaces. Human Relations, 48(3), 265-284.

Consejo Superior de Educación de Chile. (2006). Titulados de Instituciones en Licenciamiento con el CSE 1992-2006. Santiago: Autor.

Contreras, C. \& Plaza, G. (2004, agosto). Participación femenina en el mercado laboral chileno. ¿Cuánto importan los factores culturales? Encuentro 2004 de la Sociedad de Economía de Chile, Villa Alemana, Chile.

Duarte, K. (2000). ¿Juventud o juventudes? Acerca de cómo mirar y remirar las juventudes de nuestro continente. Revista Última Década, 13, 59-77.

Eagly, A. \& Carli, L. (2007). Women and the labyrinth of leadership. Harvard Business Review, September, 62-71.

Friedman, S. \& Weissbrod, C. (2005). Work and family commitment and decision-making status among emerging adults. Sex Roles, 53(5-6), 317-325.

Gaeddert, W. (1985). Sex and sex role effects on achievement strivings: Dimensions of similarity and difference. Journal of Personality, 53, 286-305. 
Good, G. \& Mintz, L. (1990). Gender role conflict and depression in college men: Evidence for compounded risk. Journal of Counseling and Development, 69, 17-21.

Kerpelman, J. L. \& Schvaneveldt, P. L. (1999). Young adult's anticipated identity importance of career, marital, and parental roles: Comparisons of men and women with different role balance orientations. Sex Roles, 41, 189-217.

Maureira, S. (2008). De lo épico a lo cotidiano: jóvenes y generaciones políticas en Chile. Revista de Ciencia Política, 28(2), 143-160.

McWhirter, E. \& McWhirter, B. (2008). Adolescent future expectations of work, education, family, and community development of a new measure. Youth Eु Society, 2(40), 182-202.

Ministerio del Trabajo de Chile. (2010). Mujer y trabajo. Recuperado el 15 de noviembre de 2010, de http:// www.subtrab.trabajo.gob.cl/?page_id=129
Niles, S. \& Goodnough, G. (1996). Life-role salience and values: A review of recent research. Career Development Quarterly, 45, 65-86.

Olavarría, J. (2001). Y todos querían ser buenos padres. Santiago: FLACSO.

Pontificia Universidad Católica de Chile \& Adimark/ GfK. (2007). Encuesta Nacional Bicentenario UCAdimark 07. Santiago: Autor.

Rapoport, R., Bailyn, L., Fletcher, J. \& Pruitt, B. (2002). Beyond work-family balance: Advancing gender equity and workplace performance. San Francisco: Jossey-Bass.

Sanders, R. (2004). Career and family priorities of college students. Disponible en http://clearinghouse.missouriwestern.edu/manuscripts/504.php

Shockley, K. M. \& Singla, N. (2011). Reconsidering work-family interactions and satisfaction: A metaanalysis. Journal of Management, 37(3), 861-886. 